\title{
Design of a modular testing platform for the handling and study of endovascular devices
}

\begin{abstract}
A design for a modular testing platform to objectively evaluate the behavior and characteristics of specialized endovascular instruments (guidewires/catheters) was presented and discussed. The platform comprises of an instrument driving mechanism and an interchangeable channel module depending on the parameter that is being measured. This platform could be used to study and benchmark commercial endovascular instruments. Such a guide would be useful to assist clinicians in the selection of the best tools for a given procedure and derive the behavioral model for each instrument.
\end{abstract}

Keyword: Endovascular devices; Instrument testing; Modeling 